\section{Avaliação da qualidade da atenção primária pelos profissionais de saúde: comparação entre diferentes tipos de serviços}

\author{
Quality assessment of primary care by health \\ professionals: a comparison of different \\ types of services
}

\begin{abstract}
1 Faculdade de Medicina, Universidade Federal do Rio Grande do Sul, Porto Alegre, Brasil.

Correspondência R. C. L. Castro

Programa de Pós-graduação em Epidemiologia, Faculdade de Medicina, Universidade Federal do Rio Grande do Sul. Rua Ramiro Barcelos 2400, 2 o andar, Porto Alegre, $R S$ 90035-003, Brasil. rodrigo_caprio@yahoo. com.br
\end{abstract}

Rodrigo Caprio Leite de Castro 1

Daniela Riva Knauth 1

Erno Harzheim 1

Lisiane Hauser 1

Bruce Bartholow Duncan 1

\section{Introdução}

In Porto Alegre, Rio Grande do Sul State, Brazil, public primary healthcare $(\mathrm{PHC})$ is provided by traditional Basic Health Units (BHU), the Family Health Strategy (FHS), the Murialdo Health Center (MHC), and the Community Health Service (CHS) of Grupo Hospitalar Conceição. This study compares the quality of adult healthcare among these different types of services. Quality of care was measured by interviews focusing on the experience of physicians and nurses in the services ( $n=340$ ), using the Primary Care Assessment Tool. Overall scores for the FHS, MHC, and CHS were significantly higher than for the BHU. The CHS also showed a significantly higher overall score compared to the FHS and MHC. In the multivariate analysis, the variables "specialized training in PHC" and "continuing education" were associated with high overall score. Thus, expansion of FHS coverage and investment in professional training are important potential strategies for improving PHC in Porto Alegre.

Health Services Evaluation; Quality of Health Care; Primary Health Care
Segundo a Organização Pan-Americana da Saúde (OPAS) 1, a Atenção Primária à Saúde (APS) é uma estratégia para organizar os Sistemas de Saúde, de maneira a possibilitar o "acesso universal aos serviços" e a "atenção integral e integrada ao longo do tempo”. O número de estudos evidenciando o impacto positivo da APS sobre os sistemas de saúde aumentou muito a partir da década de 1990. A literatura científica apresenta evidências sobre a associação entre o maior grau de orientação à APS e o aumento da efetividade dos sistemas de saúde, da promoção da equidade, da satisfação dos usuários e da eficiência 2. Embora a maior parte dos estudos tenha sido realizada em países desenvolvidos, existem também evidências do impacto positivo da APS sobre os sistemas de saúde dos países em desenvolvimento ${ }^{3}$, especialmente em países da América Latina 4.

No Brasil, a estratégia adotada pelo Ministério da Saúde para a expansão da APS e, portanto, para a reorientação do Sistema Único de Saúde (SUS) é a Estratégia Saúde da Família (ESF), iniciada em 1994 5. O financiamento para expansão da ESF vem crescendo, assim como o número de equipes implantadas; em outubro de 2010 , já eram 39.710 equipes, acompanhando 52,1\% da população brasileira (Ministério da Saúde. http://200.214.130.35/dab/historico_cobertura_ sf.php, acessado em 14/Dez/2010). 
Da mesma forma como se observa nos estudos internacionais, muitas pesquisas mostram o impacto positivo da ESF sobre o sistema de saúde brasileiro. Existem evidências sobre a associação entre a maior cobertura da ESF e indicadores como menor mortalidade infantil 6,7,8 e maior promoção da equidade 7,8. Em pesquisas comparativas entre unidades da ESF e as Unidades Básicas de Saúde tradicionais (UBS), vinculadas aos Estudos de Linha de Base (ELB) do Projeto de Expansão e Consolidação da ESF (PROESF), realizadas em municípios do Nordeste e do Sul, mostrou-se maior adequação da ESF quanto à oferta e à utilização de ações de saúde 9 , além de maior qualidade da atenção ao pré-natal e à puericultura 10 , embora estivessem em um contexto de baixa efetividade de ambos os modelos. Por outro lado, estudos vinculados aos ELB, realizados em municípios do Nordeste, evidenciaram grande heterogeneidade no impacto da ESF sobre indicadores de saúde 11 e também sobre as práticas de cuidado 12 .

Para a realização do presente estudo, utilizouse o conceito de APS proposto por Starfield 13. A autora conceitua a APS por meio de quatro atributos essenciais: o acesso de primeiro contato, que é a "acessibilidade e uso do serviço a cada novo problema ou novo episódio de um problema" 13 (p. 61); a longitudinalidade, que "pressupõe a existência de uma fonte regular de atenção e seu uso ao longo do tempo" 13 (p. 62); a integralidade, que implica "fazer arranjos para que o paciente receba todos os tipos de serviços de atenção à saúde" 13 (p. 62); e a coordenação, que é a "disponibilidade de informações a respeito de problemas e serviços anteriores e o reconhecimento daquela informação na medida em que está relacionada às necessidades para o presente atendimento" 13 (p. 365-6). Além desses, Starfield também define três atributos derivados: a orientação familiar, decorrente da consideração do contexto familiar na atenção integral; a orientação comunitária, que decorre do reconhecimento das necessidades sociais; e a competência cultural, que envolve a atenção às necessidades de uma população com "características culturais especiais".

Assim, a autora conceitua um serviço de APS pela presença dos quatro atributos essenciais que, combinados, são exclusivos da atenção primária, sendo esta qualificada pela presença dos atributos derivados. Com base nessa definição, Starfield e colaboradores produziram o instrumento Primary Care Assessment Tool (PCATool), nas versões Child Consumer/Client Surveye Adult Consumer/Client Survey, aplicáveis aos usuários para avaliação da atenção à saúde da criança e do adulto respectivamente, e Facility/Provider Survey, aplicável aos profissionais de saúde, cujo objetivo é medir a presença e a extensão de cada atributo da APS 13. O instrumento produz escores para cada atributo e também os Escores Essencial e Geral, possibilitando mensurar o grau de orientação à APS em diferentes serviços e sistemas de saúde 13 .

No Brasil, foram realizados estudos utilizando a versão para profissionais de saúde do PCATool, com o objetivo de avaliar serviços de APS e comparar unidades da ESF com as UBS. Evidenciou-se, que, de maneira geral, conforme a experiência dos profissionais, a ESF apresenta melhor estrutura e processo de atenção em comparação com as UBS 14,15,16,17,18.

O presente estudo tem por objetivo comparar a qualidade da atenção à saúde do adulto entre os diferentes tipos de serviços de APS de Porto Alegre, Rio Grande do Sul, medida por meio do instrumento PCATool, versão para profissionais de saúde.

\section{Metodologia}

Trata-se de estudo transversal, realizado com profissionais médicos e enfermeiros das unidades de saúde amostradas nos diferentes tipos de serviços públicos de APS de Porto Alegre, no período compreendido entre julho de 2006 e agosto de 2007.

Em Porto Alegre, a rede pública de APS é constituída por diferentes tipos de serviços: UBS, ESF, ambas sob a responsabilidade da Secretaria Municipal de Saúde; Centro de Saúde-Escola Murialdo (CSEM), vinculado à Secretaria Estadual de Saúde; e Serviço de Saúde Comunitária (SSC) do Hospital Nossa Senhora da Conceição, integrante do Grupo Hospitalar Conceição, uma instituição pública federal. No que se refere à cobertura populacional de cada serviço, é possível afirmar, de maneira aproximada, que a ESF, segundo dados do Departamento de Atenção Básica do Ministério da Saúde (http://dab.sau de.gov.br/historico_cobertura_sf.php, acessado em 12/Ago/2009), cobre $22 \%$ da população do município, enquanto o CSEM cobre $4 \%$, correspondendo a 52 mil pessoas 19; o SSC cobre 9\%, correspondendo a 120 mil pessoas 20 , e as UBS cobrem, aproximadamente, $65 \%$ da população de Porto Alegre. Essas proporções permanecem, de modo geral, inalteradas desde o período de realização da presente pesquisa. As diferenças entre os quatro serviços de APS avaliados estão descritas na Tabela 1.

O presente estudo está inserido no projeto A Avaliação da Qualidade do Processo de Atenção e da sua Efetividade sobre a Saúde do Adulto no Programa Saúde da Família e em Modelos 
Tabela 1

Diferenças entre os serviços de atenção primária à saúde de Porto Alegre, Rio Grande do Sul, Brasil.

\begin{tabular}{|c|c|c|c|c|}
\hline & UBS & ESF & CSEM & SSC \\
\hline $\begin{array}{l}\text { Características } \\
\text { gerais das equipes }\end{array}$ & $\begin{array}{c}\text { Multiprofissionais e } \\
\text { heterogêneas com relação } \\
\text { ao número e à categoria } \\
\text { profissional }\end{array}$ & $\begin{array}{c}\text { Multiprofissionais e } \\
\text { homogêneas com relação } \\
\text { ao número e à categoria } \\
\text { profissional }\end{array}$ & $\begin{array}{c}\text { Multiprofissionais e } \\
\text { heterogêneas com relação } \\
\text { ao número e à categoria } \\
\text { profissional }\end{array}$ & $\begin{array}{c}\text { Multiprofissionais e } \\
\text { heterogêneas com relação } \\
\text { ao número e à categoria } \\
\text { profissional }\end{array}$ \\
\hline $\begin{array}{l}\text { Composição da } \\
\text { equipe }\end{array}$ & $\begin{array}{c}\text { De maneira geral são } \\
\text { constituídas por médicos } \\
\text { internistas, pediatras, } \\
\text { ginecologistas, enfermeiros } \\
\text { e odontólogos, podendo } \\
\text { contar ainda com psicólogos, } \\
\text { assistentes sociais, entre } \\
\text { outros. Não possui ACS }\end{array}$ & $\begin{array}{l}\text { Médico, enfermeiro, } \\
\text { ACS. Algumas unidades } \\
\text { possuem odontólogos. } \\
\text { Médicos e enfermeiros de } \\
\text { qualquer especialidade } \\
\text { podem ser contratados, } \\
\text { inclusive profissionais sem } \\
\text { especialidade }\end{array}$ & $\begin{array}{c}\text { MFC, enfermeiros, } \\
\text { podendo contar ainda com } \\
\text { psicólogos, assistentes } \\
\text { sociais, nutricionistas, } \\
\text { fisioterapeutas, odontólogos. } \\
\text { Médicos internistas, } \\
\text { pediatras, ginecologistas e } \\
\text { psiquiatras realizam apoio } \\
\text { matricial. Não possui ACS }\end{array}$ & $\begin{array}{c}\text { MFC, enfermeiros, ACS, } \\
\text { podendo contar ainda com } \\
\text { psicólogos, assistentes } \\
\text { sociais, nutricionistas, } \\
\text { terapeutas ocupacionais, } \\
\text { farmacêuticos, odontólogos. } \\
\text { Médicos internistas, } \\
\text { pediatras e psiquiatras } \\
\text { realizam apoio matricial }\end{array}$ \\
\hline $\begin{array}{l}\text { Acesso a exames } \\
\text { complementares }\end{array}$ & $\begin{array}{l}\text { Laboratoriais básicos, ECG, } \\
\text { radiografias e mamografias }\end{array}$ & $\begin{array}{l}\text { Laboratoriais básicos, ECG, } \\
\text { radiografias e mamografias }\end{array}$ & $\begin{array}{c}\text { Exames laboratoriais básicos } \\
\text { possuem cota pela SMS e } \\
\text { uma cota complementar pela } \\
\text { SES. Exames de radiografia } \\
\text { e mamografia possuem cota } \\
\text { pela SMS. Existe acesso } \\
\text { direto a endoscopias }\end{array}$ & $\begin{array}{c}\text { Exames laboratoriais } \\
\text { básicos e os de imagem } \\
\text { são realizados no HNSC. } \\
\text { Possui acesso direto a } \\
\text { eletrocardiograma de } \\
\text { esforço, endoscopia e } \\
\text { exames de medicina } \\
\text { nuclear. Exames de TC } \\
\text { e RM possuem cota pré- } \\
\text { determinada para o serviço }\end{array}$ \\
\hline $\begin{array}{l}\text { Acesso à atenção } \\
\text { secundária }\end{array}$ & $\begin{array}{l}\text { Agendamento por meio da } \\
\text { Central de Marcação da SMS }\end{array}$ & $\begin{array}{l}\text { Agendamento por meio da } \\
\text { Central de Marcação da SMS }\end{array}$ & $\begin{array}{l}\text { Agendamento por meio } \\
\text { da Central de Marcação da } \\
\text { SMS. Possui acesso direto } \\
\text { à oftalmologia (refração), } \\
\text { cardiologia, dermatologia, } \\
\text { pneumologia, nefrologia e } \\
\text { colposcopia }\end{array}$ & $\begin{array}{l}\text { Agendamento por meio } \\
\text { da Central de Marcação da } \\
\text { SMS. Possui acesso direto } \\
\text { à patologia cervical, à } \\
\text { mastologia e ao ambulatório } \\
\text { de pré-natal de alto risco }\end{array}$ \\
\hline $\begin{array}{l}\text { Acesso a leito } \\
\text { hospitalar }\end{array}$ & $\begin{array}{l}\text { Via serviços de emergência. } \\
\text { Sem acesso direto }\end{array}$ & $\begin{array}{l}\text { Via serviços de emergência. } \\
\text { Sem acesso direto }\end{array}$ & $\begin{array}{l}\text { Via serviços de emergência. } \\
\text { Sem acesso direto }\end{array}$ & $\begin{array}{c}\text { Via serviços de emergência. } \\
\text { Possui acesso direto à } \\
\text { internação através de } \\
\text { enfermaria no HNSC com } \\
30 \text { leitos }\end{array}$ \\
\hline
\end{tabular}

ACS: agente comunitário de saúde; CSEM: Centro Saúde Escola Murialdo; ECG: eletrocardiograma em repouso; ESF: Estratégia Saúde da Família; HNSC: Hospital Nossa Senhora da Conceição; MFC: médicos de família e comunidade; RM: ressonância magnética; SES: Secretaria Estadual de Saúde do Rio Grande do Sul; SMS: Secretaria Municipal de Saúde de Porto Alegre; SSC: Serviço de Saúde Comunitária do Grupo Hospitalar Conceição;

TC: tomografia computadorizada; UBS: Unidades Básicas de Saúde tradicionais.

Alternativos na Rede de Atenção Primária do $\mathrm{Mu}$ nicípio de Porto Alegre 21. Para proceder a essa avaliação, o projeto utilizou a experiência de usuários, profissionais de saúde e gestores com os serviços e também os resultados alcançados.
Foram selecionados, aleatoriamente, 50\% das UBS e das unidades da ESF de cada Gerência Distrital do município, totalizando, respectivamente, 26 e 31 unidades desses dois serviços. O CSEM e o SSC são compostos por 7 e 12 unidades 
respectivamente e, em ambos, todas as unidades foram selecionadas. Esse amplo número de unidades selecionadas objetivou garantir heterogeneidade amostral.

A amostra de profissionais de saúde analisada foi constituída por todos os médicos e enfermeiros que, nas unidades selecionadas, atendiam adultos e cumpriam carga-horária $\geq 20$ horas semanais; consistiu, portanto, em uma amostragem por conveniência. Foram excluídos os profissionais que necessitavam obrigatoriamente de encaminhamento (referência) para atender. A amostra foi constituída por 181 profissionais nas UBS, 88 nas unidades da ESF, 23 no CSEM e 77 no SSC.

A qualidade da atenção à saúde está sendo entendida, no presente estudo, como a presença e a extensão dos atributos considerados essenciais e derivados da APS, de acordo com Starfield 13 . Para a sua medida, foi utilizado o instrumento PCATool, versão para profissionais de saúde 22 . O PCATool mede a presença e a extensão de cada atributo da APS por meio de escala Likert ("com certeza sim", "provavelmente sim", "provavelmente não”, “com certeza não” e "não sei/ não lembro”), possibilitando construir escores no intervalo de 1 a 4 para cada atributo. Para fins de análise, as respostas marcadas em "não sei/ não lembro" foram consideradas "provavelmente não" 22 .

Foram constituídos escores para os atributos essenciais, "acesso de primeiro contato", "longitudinalidade”, “coordenação”, “integralidade”, e para os atributos derivados, "orientação familiar” e "orientação comunitária”. O instrumento avalia a estrutura e o processo de atenção a respeito de cada atributo da APS em um determinado serviço 13 , porém, com relação à "coordenação", foram obtidos escores para os seus componentes separadamente, de maneira que o escore para "coordenação sistemas de informações" avaliou a estrutura envolvida com esse atributo, enquanto o escore para "coordenação do cuidado", considerou o processo. Da mesma forma, o escore para "integralidade serviços disponíveis" mediu a estrutura dos serviços envolvida com esse atributo, e o escore para "integralidade serviços prestados”, o processo. Os escores foram obtidos pela média aritmética dos itens que os compõem. Da mesma forma, foram constituídos os Escores Essencial e Geral da APS, obtidos, respectivamente, pela média aritmética dos escores dos atributos essenciais e dos escores de todos os atributos 22 .

A fim de caracterizar os profissionais entrevistados, utilizou-se um questionário estruturado com questões sociodemográficas, relativas à formação acadêmica e à situação ocupacional.
A satisfação dos profissionais foi avaliada em relação ao trabalho no serviço de saúde, ao apoio prestado pelo coordenador da equipe, ao relacionamento entre os membros da equipe e ao relacionamento entre a comunidade e o serviço de saúde. Também se perguntou sobre a satisfação com a abordagem feita, pelo serviço, aos pacientes portadores de diabetes, hipertensão e cardiopatia isquêmica. Para a medida da satisfação, foi utilizada escala Likert: cartão com faces expressando diferentes graus de satisfação, do maior (letra A) ao menor (letra E). Para fins de análise, foi considerado "satisfeito" o entrevistado que assinalou os dois primeiros itens da escala Likert; foi considerado "insatisfeito" o que assinalou os três últimos itens da escala, para qualquer uma das perguntas.

Todas as entrevistas foram agendadas e realizadas por entrevistadores treinados no local de trabalho dos profissionais. O treinamento e o acompanhamento sistemático dos entrevistadores foram realizados pelo mesmo coordenador de campo.

Os questionários foram escaneados e as imagens, após verificação, convertidas para uma base de dados por meio do software Teleform (Autonomy, an HP Company, Vista, Estados Unidos). Inicialmente, todos os profissionais entrevistados tiveram os escores dos atributos da APS e também os Escores Essencial e Geral calculados. Esses escores, originalmente variando em escala de 1 a 4, foram transformados em escala de 0 a 10 , mediante a seguinte fórmula: escore de $0 \mathrm{a}$ 10 do atributo $\mathrm{X}=$ (escore de 1 a 4 do atributo $\mathrm{X}$ - 1) X 10 / (4 - 1). O escore igual a ou maior de 6,6 foi considerado alto escore de APS. Esse valor foi escolhido por corresponder, na escala de 1 a 4, ao escore 3 ("provavelmente sim") 22.

Para todas as análises estatísticas, foi adotado o nível de significância de 5\%. Os escores médios de cada atributo da APS por tipo de serviço foram estimados por meio de modelo misto 23 , a fim de considerar as características da amostra, na qual as unidades de saúde constituem um subagrupamento de cada tipo de serviço. Nesse modelo, considerou-se o tipo de serviço, o efeito fixo, e as unidades de saúde dentro de cada tipo de serviço, o efeito aleatório. O efeito fixo foi a variável que representou os quatro tipos de serviços, para os quais se deseja fazer inferências, e o efeito aleatório, as unidades de saúde que constituem uma amostra da população de unidades. Dessa forma, considera-se a variabilidade das informações dentro das unidades e também entre as unidades para estimar o efeito do tipo de serviço.

No caso da suposição de dados com distribuição normal não satisfeita (o componente "coordenação sistemas de informações” e o atributo 
“orientação familiar”), optou-se pela metodologia de modelo linear generalizado misto, que permite os ajustes de efeito fixo com dados que não sejam de distribuição normal e também permite incorporar efeitos aleatórios. Para a comparação dos escores de APS entre os quatro serviços avaliados, foi utilizado o teste de comparação múltipla de Tukey. Essas análises foram realizadas por intermédiio do software SAS 9.1 (SAS Inst., Cary, Estados Unidos), utilizando os procedimentos Mixed e Glimmix.

Utilizou-se o teste qui-quadrado de Pearson para comparação entre proporções, seguido, quando necessário, do teste de comparação múltipla. Além disso, para a identificação das variáveis que, no nível do profissional, estão associadas com o alto Escore Geral da APS, utilizou-se a regressão de Poisson com variância robusta, sendo apresentada como medida de efeito a razão de prevalência (RP) com seus intervalos de confiança (IC). No modelo multivariável, foram incluídas, inicialmente, as variáveis independentes que se mostraram, individualmente, associadas ao alto Escore Geral de APS com valor de $\mathrm{p}<0,20$. Posteriormente, foram sendo excluídas as variáveis com os maiores valores de $\mathrm{p}$, de maneira que permaneceram, no modelo final, apenas aquelas com valores de $\mathrm{p}<0,05$. Essas análises foram realizadas no Stata 9 (Stata Corp., College Station, Estados Unidos), utilizando-se os comandos escritos pelo menu survey (svy), usados para o controle do efeito de cluster, por meio do método de linearização de Taylor. Dessa maneira, foi possível incorporar correções nas estimativas de variabilidade, permitindo a adequação da análise ao plano amostral.

O projeto foi aprovado pelos Comitês de Ética em Pesquisa de todas as instituições envolvidas no estudo. Somente foram realizadas entrevistas com os profissionais que aceitaram participar e assinaram o Termo de Consentimento Livre e Esclarecido.

\section{Resultados}

Do total previsto de 369 profissionais médicos e enfermeiros incluídos, foram realizadas 340 entrevistas (226 médicos e 114 enfermeiros), verificando-se 7,9\% de perdas (29 profissionais). Foi considerada "perda" a entrevista não realizada por razão de recusa, troca de unidade, afastamento por motivo de saúde ou demissão. Nas UBS, registraram-se $11 \%$ de perdas; nas unidades da ESF, 3,4\%; no CSEM, 4,3\%; no SSC, 6,5\%.

Na Tabela 2, é apresentado o perfil sociodemográfico, acadêmico e ocupacional dos profissionais, por tipo de serviço.
A Tabela 3 apresenta os escores dos atributos e os Escores Essencial e Geral da APS para os quatro tipos de serviços avaliados, sendo destacadas as diferenças significativas encontradas entre os escores das unidades da ESF, do CSEM e do SSC, quando comparados aos das UBS. Além dos resultados apresentados nesta tabela, é importante observar que o SSC também mostrou Escores Essencial e Geral significativamente maiores do que os atribuídos à ESF e ao CSEM. Com relação aos atributos, comparando-se SSC e ESF, observouse diferença significativa na "longitudinalidade", "integralidade serviços disponíveis" e "orientação comunitária” a favor do SSC; comparando-se SSC e CSEM, houve diferença significativa na "integralidade serviços disponíveis" e "orientação comunitária”, também a favor do SSC. A respeito da comparação entre CSEM e ESF, notou-se diferença significativa somente na "integralidade serviços disponíveis” a favor do CSEM.

As proporções de profissionais que atribuíram baixo e alto Escore, Essencial e Geral, de APS por tipo de serviço, mediante escores categorizados, encontram-se na Tabela 4. No SSC, podem ser observadas as maiores proporções de alto Escore Essencial e Geral de APS atribuídos, enquanto as menores encontram-se nas UBS. Embora as unidades da ESF, em comparação com CSEM, tenham mostrado maiores proporções de alto Escore Essencial e Geral de APS, os valores são bastante próximos.

Na Tabela 5, podemos observar as variáveis que apresentaram, no modelo univariável, valor de $\mathrm{p}$ menor do que o estabelecido como ponto de corte (valor de $\mathrm{p}<0,20$ ). As variáveis "sexo", "categoria profissional”, se o entrevistado "possui especialidade", "tempo de trabalho no serviço de saúde (em anos)", "tempo de trabalho na unidade de saúde (em anos)", "número médio de consultas semanais atendidas no serviço de saúde" e se o entrevistado é "coordenador(a) da unidade" apresentaram valor de $\mathrm{p}$ maior do que o estabelecido como ponto de corte.

Entre as variáveis "tempo de formado" e "idade”, verificou-se correlação ( $r=0,90$, valor de $\mathrm{p}<$ 0,001 ), optando-se por incluir, no modelo, apenas o "tempo de formado", pois esteve associado com a prevalência de alto Escore Geral atribuído por profissionais em estudo de Cunha et al. 15. Como resultado do modelo multivariável, pôde-se verificar que apenas as variáveis "especialidade na área de APS" e "oferta de educação continuada pelo serviço" associaram-se com o aumento da prevalência de alto Escore Geral atribuído, pelos profissionais, à atenção à saúde do adulto.

Na Tabela 6, são apresentadas as proporções de profissionais médicos e enfermeiros satisfeitos com o trabalho, com o coordenador, com o 
Perfil dos profissionais médicos e enfermeiros por tipo de serviço de atenção primária de Porto Alegre, Rio Grande do Sul, Brasil.

\begin{tabular}{|c|c|c|c|c|c|c|c|c|}
\hline \multirow[t]{2}{*}{ Variáveis } & \multicolumn{2}{|c|}{ UBS $(n=161)$} & \multicolumn{2}{|c|}{$\operatorname{ESF}(n=85)$} & \multicolumn{2}{|c|}{$\operatorname{CSEM}(n=22)$} & \multicolumn{2}{|c|}{$\operatorname{SSC}(n=72)$} \\
\hline & $\%$ ou média & DP & $\%$ ou média & DP & $\%$ ou média & $\mathrm{DP}$ & $\%$ ou média & DP \\
\hline \multicolumn{9}{|l|}{ Sociodemográficas } \\
\hline Idade (anos) & 46,2 & 8,9 & 38,6 & 9,6 & 42,3 & 8,4 & 43,9 & 8,3 \\
\hline \multicolumn{9}{|l|}{ Sexo } \\
\hline Masculino & 32,9 & & 22,4 & & 13,6 & & 30,6 & \\
\hline Feminino & 67,1 & & 77,6 & & 86,4 & & 69,4 & \\
\hline \multicolumn{9}{|l|}{ Formação acadêmica } \\
\hline \multicolumn{9}{|l|}{ Graduação } \\
\hline Médico(a) & 77,0 & & 51,8 & & 68,2 & & 59,7 & \\
\hline Enfermeiro(a) & 23,0 & & 48,2 & & 31,8 & & 40,3 & \\
\hline Tempo de formado (anos) & 20,0 & 8,9 & 11,0 & 8,4 & 15,6 & 7,9 & 18,7 & 8,0 \\
\hline Possui especialidade & 90,7 & & 67,1 & & 100,0 & & 100,0 & \\
\hline \multicolumn{9}{|l|}{ Especialidade } \\
\hline Na área de APS * & 14,2 & & 33,0 & & 59,1 & & 68,0 & \\
\hline Outras & 76,5 & & 34,1 & & 40,9 & & 32,0 & \\
\hline \multicolumn{9}{|l|}{ Ocupacionais } \\
\hline Número de locais de trabalho & 2,3 & 1,0 & 1,4 & 0,7 & 2,0 & 0,7 & 1,6 & 0,7 \\
\hline $\begin{array}{l}\text { Tempo de trabalho no serviço de saúde } \\
\text { (anos) }\end{array}$ & 11,0 & 8,7 & 3,3 & 2,8 & 11,2 & 7,4 & 11,8 & 7,7 \\
\hline $\begin{array}{l}\text { Tempo de trabalho na unidade de saúde } \\
\text { (anos) }\end{array}$ & 8,0 & 7,9 & 1,9 & 1,7 & 6,5 & 4,1 & 8,4 & 7,4 \\
\hline Carga horária semanal no serviço de saúde & 29,0 & 8,2 & 38,1 & 5,7 & 27,0 & 7,7 & 34,4 & 3,8 \\
\hline $\begin{array}{l}\text { Consultas semanais atendidas no serviço de } \\
\text { saúde }\end{array}$ & 58,4 & 24,6 & 74,7 & 36,3 & 49,0 & 35,4 & 49,4 & 29,6 \\
\hline Coordenador(a) da unidade & 9,3 & & 36,5 & & 18,2 & & 9,7 & \\
\hline \multicolumn{9}{|l|}{ Educação continuada } \\
\hline Oferta de educação continuada pelo serviço & 72,0 & & 84,7 & & 72,7 & & 100,0 & \\
\hline
\end{tabular}

APS: Atenção Primária à Saúde; CSEM: Centro Saúde Escola Murialdo; ESF: Estratégia Saúde da Família; SSC: Serviço de Saúde Comunitária do Grupo Hospitalar Conceição; UBS: Unidades Básicas de Saúde tradicionais.

* Medicina de Família e Comunidade (residência ou prova de título) para médicos, ou Enfermagem Comunitária (residência ou especialização) para enfermeiros, ou Saúde da Família (especialização) para ambas as categorias.

relacionamento entre os membros da equipe e com a comunidade e, também, com a abordagem feita aos pacientes, por tipo de serviço. Observa-se que, no SSC, encontra-se a maior proporção de satisfeitos com o trabalho e, no CSEM, a menor. Quanto à satisfação com a abordagem do serviço aos pacientes portadores de diabetes, hipertensão e cardiopatia isquêmica, a ESF teve as maiores proporções de satisfeitos com as três abordagens, enquanto o CSEM apresentou as menores. As diferenças significativas encontradas na comparação entre as proporções de satisfeitos nos diferentes tipos de serviço são informadas, também, na Tabela 6 .

A associação entre a atribuição de alto Escore Geral e a satisfação com o trabalho foi verificada por meio do teste de correlação de Spearman. O resultado mostra correlação fraca, mas positiva e significativa $(r=0,27$, valor de $\mathrm{p}<0,001)$. Observou-se, igualmente, significância estatística entre a atribuição de alto Escore Geral e a satisfação com o relacionamento com a comunidade $(\mathrm{r}=0,24$, valor de $\mathrm{p}<0,001)$, com a abordagem a pacientes diabéticos $(\mathrm{r}=0,34$, valor de $\mathrm{p}<0,001)$, hipertensos $(\mathrm{r}=0,34$, valor de $\mathrm{p}<0,001)$ e cardiopatas isquêmicos $(r=0,34$, valor de $\mathrm{p}<0,001)$. Não se encontrou significância estatística na correlação entre a atribuição de alto Escore Geral e a satisfação com o coordenador $(r=0,08$, valor de $\mathrm{p}=0,173$ ) e com o relacionamento entre os membros da equipe $(r=0,09$, valor de $p=0,086)$. 
Comparação dos escores dos atributos da Atenção Primária à Saúde (APS), atribuídos pelos profissionais médicos e enfermeiros, entre os serviços de atenção primária de Porto Alegre, Rio Grande do Sul, Brasil.

\begin{tabular}{|c|c|c|c|c|}
\hline \multirow[t]{2}{*}{ Atributos da APS } & \multicolumn{4}{|c|}{ Escores (0-10) } \\
\hline & UBS $(n=161)$ & $\operatorname{ESF}(n=85)$ & $\operatorname{CSEM}(n=22)$ & $\operatorname{SSC}(n=72)$ \\
\hline Acesso de primeiro contato & 3,14 & 3,53 * & 2,92 & 3,52 \\
\hline Longitudinalidade & 6,21 & 6,53 & 6,68 & 7,27 * \\
\hline Integralidade (serviços disponíveis) & 6,29 & 6,50 & $7,29 *$ & 8,30 * \\
\hline Integralidade (serviços prestados) & 7,02 & 8,27 * & 8,35 * & 8,49 * \\
\hline Coordenação do cuidado & 6,92 & 7,17 & 6,59 & 7,45 * \\
\hline Coordenação (sistemas de informações) & 9,00 & 9,02 & 9,04 & 9,15 \\
\hline Orientação familiar & 8,21 & 8,87 * & 8,83 & 9,47 * \\
\hline Orientação comunitária & 5,58 & 6,75 * & 7,42 * & 8,73 * \\
\hline Escore essencial de APS ** & 6,45 & 6,84 * & 6,80 & 7,36 * \\
\hline Escore geral da APS *** & 6,58 & 7,08 * & 7,13 * & 7,80 * \\
\hline
\end{tabular}

CSEM: Centro Saúde Escola Murialdo; ESF: Estratégia Saúde da Família; SSC: Serviço de Saúde Comunitária do Grupo Hospitalar Conceição; UBS: Unidades Básicas de Saúde tradicionais.

* Significância pelo teste de Tukey, em comparação com UBS, valor de $p<0,05$;

** Estimado por meio de modelo misto considerando os escores dos atributos essenciais;

*** Estimado por meio de modelo misto considerando os escores de todos os atributos.

Tabela 4

Proporção de profissionais médicos e enfermeiros que atribuíram baixo e alto Escore, Essencial e Geral, por tipo de serviço de Atenção Primária à Saúde (APS) de Porto Alegre, Rio Grande do Sul, Brasil.

\begin{tabular}{|c|c|c|c|c|c|c|c|c|}
\hline \multirow[t]{3}{*}{ Serviços de APS } & \multicolumn{4}{|c|}{ Escore essencial da APS * } & \multicolumn{4}{|c|}{ Escore geral da APS * } \\
\hline & \multicolumn{2}{|c|}{ Baixo } & \multicolumn{2}{|c|}{ Alto } & \multicolumn{2}{|c|}{ Baixo } & \multicolumn{2}{|c|}{ Alto } \\
\hline & $\mathrm{n}$ & $\%$ & $\mathbf{n}$ & $\%$ & $\mathrm{n}$ & $\%$ & $\mathbf{n}$ & $\%$ \\
\hline UBS $(n=161)$ & 90 & 55,9 & 71 & 44,1 & 78 & 48,4 & 83 & 51,6 \\
\hline $\operatorname{ESF}(n=85)$ & 29 & 34,1 & 56 & 65,9 & 25 & 29,4 & 60 & 70,6 \\
\hline $\operatorname{CSEM}(n=22)$ & 9 & 40,9 & 13 & 59,1 & 7 & 31,8 & 15 & 68,2 \\
\hline $\operatorname{SSC}(n=72)$ & 7 & 9,7 & 65 & 90,3 & 2 & 2,8 & 70 & 97,2 \\
\hline Valor de $p$ ** & \multicolumn{6}{|c|}{$<0,001$} & \multicolumn{2}{|c|}{$<0,001$} \\
\hline
\end{tabular}

CSEM: Centro Saúde Escola Murialdo; ESF: Estratégia Saúde da Família; SSC: Serviço de Saúde Comunitária do Grupo Hospitalar Conceição; UBS: Unidades Básicas de Saúde tradicionais.

* O escore de APS $\geq 6,6$, em uma escala de 0 a 10, é considerado alto escore;

** Teste $\chi^{2}$ de Pearson.

\section{Discussão}

Os resultados apresentados evidenciam que, na experiência dos profissionais médicos e enfermeiros, a qualidade da atenção à saúde do adulto é diferente entre os serviços de APS de Porto Alegre. Conforme a experiência dos profissionais, as UBS apresentam o menor grau de orientação à APS, o SSC, o maior; não há diferença entre o grau de orientação à APS entre CSEM e ESF.
O SSC, em comparação aos outros três serviços avaliados, mostrou, na maioria dos atributos, os maiores escores, resultando em Escores Essencial e Geral significativamente superiores aos das UBS, das unidades da ESF e do CSEM. Este último também mostrou Escore Geral significativamente maior ao atribuído às UBS, contudo não se evidenciou diferença significativa com relação à ESF. Cabe destacar que, embora o SSC e o CSEM sejam serviços pioneiros no país e centros forma- 
Variáveis associadas ao alto Escore Geral da Atenção Primária à Saúde (APS) atribuído pelos profissionais médicos e enfermeiros à atenção à saúde do adulto.

\begin{tabular}{|c|c|c|c|c|c|c|}
\hline \multirow[t]{2}{*}{ Variáveis } & \multicolumn{3}{|c|}{ Modelo univariável * } & \multicolumn{3}{|c|}{ Modelo multivariável * } \\
\hline & RP & IC95\% & Valor de $p$ & RP & IC95\% & Valor de $p$ \\
\hline \multicolumn{7}{|l|}{ Sociodemográficas } \\
\hline Idade ** & 0,99 & $0,98-1,00$ & 0,056 & & & \\
\hline \multicolumn{7}{|l|}{ Formação acadêmica } \\
\hline Tempo de formado & 0,99 & $0,98-1,00$ & 0,033 & & & \\
\hline \multicolumn{7}{|l|}{ Especialidade } \\
\hline Outras/Não possui & 1,00 & & & & & \\
\hline 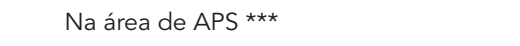 & 1,36 & $1,17-1,58$ & $<0,001$ & 1,30 & $1,14-1,49$ & $<0,001$ \\
\hline \multicolumn{7}{|l|}{ Ocupacionais } \\
\hline Número de locais de trabalho & 0,88 & $0,81-0,96$ & 0,003 & & & \\
\hline Carga horária semanal no serviço de saúde & 1,01 & $1,01-1,02$ & 0,003 & & & \\
\hline \multicolumn{7}{|l|}{ Educação continuada } \\
\hline \multicolumn{7}{|l|}{ Oferta de educação continuada pelo serviço } \\
\hline Não/Não sei & 1,00 & & & & & \\
\hline Sim & 1,80 & $1,27-2,55$ & 0,001 & 1,73 & $1,22-2,46$ & 0,002 \\
\hline
\end{tabular}

IC95\%: intervalo de 95\% de confiança; RP: razão de prevalência.

* Regressão de Poisson com variância robusta. No modelo univariável são apresentadas as variáveis com valor de p menor do que o estabelecido como ponto de corte $(p<0,20)$;

** Não incluída no modelo multivariável por apresentar correlação com "tempo de formado" $(r=0,90, p<0,001)$;

*** Medicina de Família e Comunidade (residência ou prova de título) para médicos, ou Enfermagem Comunitária (residência ou especialização) para enfermeiros, ou Saúde da Família (especialização) para ambas as categorias.

Tabela 6

Proporção de profissionais médicos e enfermeiros satisfeitos com o trabalho e com a abordagem aos pacientes, por tipo de serviço de Atenção Primária à Saúde (APS) de Porto Alegre, Rio Grande do Sul, Brasil.

\begin{tabular}{|c|c|c|c|c|c|c|c|c|c|c|c|c|c|c|}
\hline \multirow[t]{3}{*}{ Serviços de APS } & \multicolumn{8}{|c|}{ Satisfação com o(a) * } & \multicolumn{6}{|c|}{$\begin{array}{c}\text { Satisfação com a abordagem aos pacientes } \\
\text { portadores de * }\end{array}$} \\
\hline & \multicolumn{2}{|c|}{ Trabalho ** } & \multicolumn{2}{|c|}{ Coordenador } & \multicolumn{2}{|c|}{ Equipe } & \multicolumn{2}{|c|}{ Comunidade } & \multicolumn{2}{|c|}{$\begin{array}{l}\text { Diabetes } \\
\star \star \star \star\end{array}$} & \multicolumn{2}{|c|}{ Hipertensão \# } & \multicolumn{2}{|c|}{ Cardiopatia } \\
\hline & $\mathrm{n}$ & $\%$ & $\mathrm{n}$ & $\%$ & $\mathrm{n}$ & $\%$ & $\mathrm{n}$ & $\%$ & $\mathrm{n}$ & $\%$ & $\mathrm{n}$ & $\%$ & $\mathrm{n}$ & $\%$ \\
\hline UBS $(n=161)$ & 101 & 62,7 & 110 & 75,3 & 128 & 79,5 & 117 & 72,7 & 97 & 60,6 & 111 & 69,4 & 86 & 53,8 \\
\hline $\operatorname{ESF}(n=85)$ & 66 & 77,7 & 47 & 87,0 & 73 & 85,9 & 65 & 76,5 & 70 & 82,4 & 72 & 84,7 & 60 & 70,6 \\
\hline $\operatorname{CSEM}(n=22)$ & 9 & 40,9 & 15 & 83,3 & 18 & 81,8 & 14 & 63,6 & 11 & 50,0 & 13 & 59,1 & 11 & 50,0 \\
\hline $\operatorname{SSC}(n=72)$ & 63 & 87,5 & 57 & 87,7 & 61 & 84,7 & 58 & 80,6 & 52 & 73,2 & 57 & 80,3 & 50 & 70,4 \\
\hline Valor de $\mathrm{p}$ \#\# & \multicolumn{2}{|c|}{$<0,001$} & \multicolumn{2}{|c|}{0,142} & \multicolumn{2}{|c|}{0,683} & \multicolumn{2}{|c|}{0,614} & \multicolumn{2}{|c|}{0,014} & \multicolumn{2}{|c|}{0,024} & \multicolumn{2}{|c|}{0,063} \\
\hline
\end{tabular}

CSEM: Centro Saúde Escola Murialdo; ESF: Estratégia Saúde da Família; SSC: Serviço de Saúde Comunitária do Grupo Hospitalar Conceição; UBS: Unidades Básicas de Saúde tradicionais.

* Proporção de respostas A e B (satisfeitos) em uma escala Likert de cinco faces;

** SSC difere de UBS e CSEM, ESF difere de CSEM, valor de $p<0,05$;

*** ESF difere de UBS e CSEM, valor de $p<0,05$;

\# ESF difere de UBS, valor de $p<0,05$;

\#\# Teste $\chi^{2}$ de Pearson. 
dores de recursos humanos para APS, o segundo encontra-se em processo de municipalização, iniciado antes do trabalho de campo do presente estudo, e continua em trâmite; portanto, a conversão das unidades do CSEM em ESF pode explicar a não diferenciação entre estes dois tipos de serviço. De fato, na experiência dos profissionais, CSEM e ESF apresentam o mesmo grau de orientação à APS por não possuírem diferença significativa seja nos Escores Essencial e Geral, seja em qualquer atributo, com exceção para a “integralidade serviços disponíveis".

Quanto ao perfil dos profissionais, é importante destacar que, no que se refere à formação acadêmica, todos os profissionais no SSC e no CSEM possuem uma especialidade; ressalte-se, ainda, que esses serviços apresentam as maiores proporções de especialistas na área de APS, $68 \%$ e $59,1 \%$ respectivamente (neste estudo, são considerados os médicos com residência ou prova de título em Medicina de Família e Comunidade ou especialização em Saúde da Família, bem como enfermeiros com residência ou especialização em Enfermagem Comunitária ou especialização em Saúde da Família). Sobre a oferta de educação continuada, também se destaca o SSC, com $100 \%$ dos profissionais referindo essa oferta.

A ESF apresentou Escores Essencial e Geral significativamente maiores do que os atribuídos às UBS. Em todos os atributos, a primeira também obteve maiores escores do que a segunda, mas, assim como nos estudos de Chomatas 14 , Ibañez et al. 16 e van Stralen et al. ${ }^{18}$, a ESF apresentou escores maiores e estatisticamente significativos em "acesso de primeiro contato", "integralidade serviços prestados", "orientação familiar" e "orientação comunitária”. No entanto, a diferença relacionada ao atributo "longitudinalidade" a favor da ESF, também evidenciada nas pesquisas referidas e no estudo de Macinko et al. 17, não foi encontrada no presente estudo. Da mesma forma, Cunha et al. 15, em estudo comparativo entre ESF e UBS, realizado no sul de Porto Alegre, para avaliação da atenção à saúde infantil, evidenciaram, na experiência dos profissionais, diferença significativa nos escores "acesso de primeiro contato", "orientação familiar" e "orientação comunitária" a favor da ESF. No referido estudo, entretanto, não se observou diferença significativa na "integralidade" a favor da ESF.

Importante observar que, na ESF, o perfil dos profissionais encontrado é, de maneira geral, diferente do que se verifica nos outros três serviços. Na ESF, os profissionais são, em média, mais jovens (mas com desvio-padrão da idade maior) e têm menos tempo de formados; há menor proporção de profissionais especialistas, menor tempo de trabalho no serviço e na unidade de saúde; a carga-horária semanal é maior, sendo igualmente maior o número de consultas semanais atendidas. Nesse serviço, os profissionais são, também, em maior proporção, coordenadores das unidades. Esse perfil é semelhante ao encontrado em estudos realizados em outros estados e regiões do Brasil 24,25. Nesses estudos, também se evidenciou que, na ESF, os médicos e enfermeiros, de maneira geral, são mais jovens, têm menos tempo de formados e menos tempo de trabalho no serviço; igualmente, observa-se baixa proporção de profissionais especialistas. Entretanto, apesar de apresentar a menor proporção de profissionais com especialidade nas diferentes áreas, em comparação com os outros três tipos de serviços avaliados, os profissionais da ESF informaram ter especialização na área de APS em proporção que alcançou o dobro da informada pelos profissionais das UBS, resultado também encontrado no estudo de Tomasi et al. 25. Destaca-se, ainda, que a ESF apresentou a segunda maior proporção de oferta referida de educação continuada.

Os atributos "integralidade" e "orientação comunitária” se mostraram mais sensíveis na identificação das diferenças entre os serviços, como indica o maior número de diferenças significativas encontradas na comparação entre os escores desses atributos. As UBS apresentaram os menores escores, enquanto os outros três serviços mostraram escores significativamente superiores aos atribuídos a ela. Considerando que o componente "integralidade serviços disponíveis" avalia a estrutura - ou seja, a variedade de atividades com as quais a Unidade de APS é capaz de lidar, tendo em vista que deveria ter capacidade para reconhecer e manejar todos os problemas de saúde comuns em sua população ; que o componente "integralidade serviços prestados” avalia o processo - isto é, o desempenho de atividades pelo profissional - e que o atributo "orientação comunitária” avalia o conhecimento do profissional acerca das necessidades de saúde da comunidade e a sua abordagem comunitária, os dados encontrados sugerem que essas características estão menos presentes nas UBS.

Por outro lado, o componente "coordenação sistemas de informações" apresentou escores altos em todos os serviços, tendo sido o único cujos escores não apresentaram diferença significativa entre os quatro serviços. Esse achado é também evidenciado em outros estudos comparativos que utilizaram o PCATool 14,15,16,17,18, versão profissionais, podendo acontecer, em parte, porque a avaliação desse componente envolve a relação da APS com outros níveis de atenção. No caso do presente estudo, por exemplo, os quatro serviços 
estão inseridos em um mesmo sistema de saúde municipal, sujeitos, em parte, às mesmas condições de relação entre os níveis de atenção.

Na análise multivariável, "possuir especialidade na área de APS" e "oferta de educação continuada" mantiveram-se significativas, elevando a prevalência de alto Escore Geral. Conforme os resultados apresentados, ter "especialidade na área de APS" aumenta em 30\% a prevalência de alto Escore Geral e referir "a oferta de educação continuada" aumenta em $73 \%$ a mesma prevalência. Em pesquisa realizada no Município de Curitiba, Chomatas 14, ao avaliar ESF e UBS por meio do PCATool, versão profissionais de saúde, evidenciou, no modelo multivariável, que possuir "formação em medicina de família ou enfermagem comunitária" também se associou com o aumento da prevalência de alto Escore Geral da APS. No que se refere à variável "oferta de educação continuada", sabemos, pela pesquisa de Kolling 26, que mediu a experiência de médicos e enfermeiros com a ESF de 32 municípios do Rio Grande do Sul, participantes do Projeto Telessaúde, através do PCATool, versão profissionais, que a capacidade autoavaliada em habilidades específicas da APS, incluindo trabalho multidisciplinar, visita domiciliar, abordagem familiar e comunitária, associou-se significativamente com alto Escore Geral de APS atribuído. Segundo os autores, os resultados encontrados sugerem que o treinamento em habilidades específicas da APS pode ser útil para atingir maior orientação dos profissionais à APS.

Podemos reconhecer limitações no presente estudo. Inicialmente, existe uma possibilidade de o pequeno tamanho amostral de profissionais na ESF e, principalmente, no CSEM ter influenciado negativamente o poder das análises comparativas referentes a esses serviços. Contudo, é importante destacar que a ESF e o CSEM mostraram Escore Geral de APS significativamente maior do que o atribuído às UBS. O instrumento PCATool, por sua vez, também apresenta limitações. A primeira é considerar, para o cálculo dos Escores Essencial e Geral, que os atributos tenham os mesmos "pesos" na medida da orientação à APS. A segunda reside em utilizar apenas a experiência dos atores envolvidos na atenção como critério avaliador, não incorporando, por exemplo, a avaliação técnica do serviço prestado. Por último, esta é uma pesquisa avaliativa, transversal, sujeita às limitações próprias desse tipo de delineamento. Uma delas é a validade externa, já que os resultados da pesquisa avaliativa de serviços devem ser discutidos considerando-se a realidade local dos serviços; outra é a impossibilidade de inferência causal em virtude de causalidade reversa. Dessa forma, por exemplo, a correlação encontrada entre a atribuição de alto Escore Geral e a satisfação com o trabalho evidencia que as duas variam juntas, não sendo possível afirmar se a atribuição de alto Escore Geral faz com que o profissional fique mais satisfeito ou se o profissional, por estar mais satisfeito, atribui alto Escore Geral à atenção prestada pelo serviço.

O presente estudo pode ser entendido como uma parte da avaliação dos serviços, estando incluída em um projeto que utiliza também a experiência de outros atores, usuários e gestores, além dos resultados alcançados. Por ter sido realizada com os profissionais das mesmas unidades avaliadas pelos usuários e ter utilizado os itens validados no instrumento aplicado a estes, oferece uma particular possibilidade de comparação entre a experiência de profissionais e usuários sobre a qualidade da atenção prestada pelos serviços. A importância de se considerar a experiência de diferentes atores é observada nos estudos que utilizam o PCATool, versão aplicada a profissionais e usuários. Por exemplo, em um estudo realizado na ESF de Amparo, São Paulo, utilizando o PCATool 27, a "orientação familiar" apresentou, na experiência dos profissionais, o segundo escore mais alto, porém, na experiência dos usuários, o escore mais baixo, observandose, portanto, em comparação com todos os outros atributos, a maior diferença entre as experiências de profissionais e usuários. Também foi evidenciado o contrário, ou seja, em três estudos $18,27,28$, o "acesso de primeiro contato" teve pior avaliação pelos profissionais de saúde.

Por essa razão, de acordo com Serapioni ${ }^{29}$, a avaliação multidimensional é um processo que resulta, muitas vezes, na identificação de conflitos e na busca de soluções por meio do envolvimento dos diferentes atores. Portanto, com base em uma contradição observada entre a experiência dos atores envolvidos, podem-se buscar caminhos para uma “área de mediação", como proposto por Atkinson 30. Segundo este autor, os conflitos entre as perspectivas de profissionais e usuários são decorrentes de divergências entre modelos explanatórios de saúde, podendo ser resolvidos mediante treinamento e educação. Em vista disso, no caso da diferença entre as experiências de profissionais e usuários sobre a "orientação familiar", observada no estudo de Pimenta et al. 27, podemos perguntar se o alto Escore Geral atribuído pelos profissionais é consequência do conhecimento, apenas teórico, que estes têm sobre a "orientação familiar" na ESF. Ou ainda, se o baixo Escore Geral atribuído pelos usuários é consequência do baixo reconhecimento que estes possam ter em relação à abordagem familiar realmente praticada no serviço, em que se utiliza metodologia não identificada por eles. 


\section{Considerações finais}

Os resultados apresentados neste estudo trazem importantes implicações para os serviços avaliados e também para as políticas públicas de atenção à saúde no município. Todos os serviços possuem, na experiência dos profissionais, atributos que precisam ser melhorados. Por exemplo, o "acesso de primeiro contato" recebeu os menores escores em todos os serviços, de maneira que investir na estrutura e no processo de atenção relativos a esse atributo deve ser, conforme a experiência dos profissionais, prioridade para a qualificação da APS no município. Da mesma forma, as variáveis possuir "especialidade na área de APS" e "oferta de educação continuada" associaram-se com a prevalência de alto Escore Geral, evidenciando, assim, que o investimento na formação profissional especializada em APS pode ser uma estratégia de qualificação da atenção em todos os serviços. A satisfação também se apresenta como um indicador relevante. Por exemplo, a baixa satisfação com o trabalho e com a abordagem aos pacientes diabéticos, hipertensos e cardiopatas isquêmicos observada no CSEM sinaliza um problema que precisa de cuidadosa atenção por parte dos gestores desse serviço.

Em relação aos serviços gerenciados pela Secretaria Municipal de Saúde, ESF e UBS, observamos que, de acordo com a experiência dos profissionais, a ESF, em comparação às UBS, apresenta significativamente maior grau de orientação à
APS. No entanto, as UBS são responsáveis pela atenção de, aproximadamente, $65 \%$ da população de Porto Alegre, o que significa que mais da metade da população do município, na perspectiva dos profissionais, recebe atenção com o menor grau de orientação à APS. Logo, a ampliação da cobertura da ESF mostra-se como uma estratégia para a qualificação da atenção. As diferenças estruturais existentes entre ESF e UBS, como, por exemplo, a atuação da ESF em áreas adscritas, em territórios menores, por meio de profissionais generalistas e de agentes comunitários de saúde, podem explicar as diferenças encontradas entre esses serviços, de maneira que as mudanças necessárias para a qualificação das UBS estão incluídas justamente na proposta de convertêlas em unidades da ESF, configurando-se, assim, uma estratégia de qualificação da atenção. Além disso, para qualificar ainda mais a atenção, é necessário investir na ESF, principalmente, conforme os resultados apresentados, na qualificação do acesso e na formação profissional, incluindo o reconhecimento dos especialistas na área de APS e a instituição de um programa de educação continuada que alcance todos os profissionais.

Por fim, pesquisas posteriores, que busquem avaliar os serviços de APS de Porto Alegre, com base na experiência de usuários e gestores, por meio de metodologias quantitativas ou qualitativas, podem contribuir para a ampliação e aprofundamento dos resultados encontrados neste estudo.

\section{Resumo}

Em Porto Alegre, Rio Grande do Sul, Brasil, a rede pública de Atenção Primária à Saúde (APS) inclui as Unidades Básicas de Saúde tradicionais (UBS), a Estratégia Saúde da Família (ESF), o Centro Saúde Escola Murialdo (CSEM) e o Serviço de Saúde Comunitária (SSC) do Grupo Hospitalar Conceição. O objetivo deste estudo é comparar a qualidade da atenção à saúde do adulto entre esses diferentes tipos de serviços. A qualidade da atenção foi medida por meio de entrevistas sobre a experiência de médicos e enfermeiros com os serviços $(n=340)$, utilizando-se, para isso, o Primary Care Assessment Tool. O Escore Geral de APS atribuido à ESF, ao CSEM e ao SSC foi significativamente maior do que o atribuído às UBS. O SSC apresentou também Escore Geral de APS significativamente maior do que o atribuído à ESF e ao CSEM. No modelo multivariável, ter "especialidade na área de APS" e referir "oferta de educação continuada" mostraram-se associados com a prevalência de alto Escore Geral. Dessa forma, a ampliação da cobertura da ESF e o investimento na formação profissional são estratégias para qualificar a APS no município.

Avaliação de Serviços de Saúde; Qualidade da Assistência à Saúde; Atenção Primária à Saúde 


\section{Colaboradores}

R. C. L. Castro participou da concepção do estudo, revisão da literatura, coordenação do trabalho de campo, construção e revisão da base de dados, análise e interpretação dos resultados, construção das tabelas e redação do texto. D. R. Knauth, E. Harzheim e B. B. Duncan participaram da concepção do estudo, análise e interpretação dos resultados e revisão do texto. L. Hauser participou da construção e revisão da base de dados, análise e interpretação dos resultados e revisão do texto.

\section{Agradecimentos}

Este artigo faz parte de um projeto financiado pela Fundação de Amparo à Pesquisa do Estado do Rio Grande do Sul (FAPERGS), edital 008/2004 (processo 04/1507.3); pelo Conselho Nacional de Desenvolvimento Científico e Tecnológico (CNPq), edital 49/2005 (processo 402466/2005-5); e pela Coordenação de Aperfeiçoamento de Pessoal de Nível Superior (CAPES), bolsa PRODOC em 2005.

\section{Referências}

1. Organización Panamericana de la Salud/Organización Mundial de la Salud. La renovación de la atención primaria de salud en las Américas. Washington DC: Organización Panamericana de la Salud/Organización Mundial de la Salud; 2007.

2. Starfield B, Shi L, Macinko J. Contribution of primary care to health systems and health. Milbank Q 2005; 83:457-502.

3. Macinko J, Starfield B, Erinosho T. The impact of primary healthcare on population health in low and middle income countries. J Ambul Care Manage 2009; 32:150-71.

4. Moore D, Castillo E, Richardson C, Reid R. Determinants of health status and the influence of primary health care services in Latin America, 199098. Int J Health Plann Manage 2003; 18:279-92.
5. Ministério da Saúde. Saúde da família: uma estratégia para a reorientação do modelo assistencial. Brasília: Ministério da Saúde; 1998.

6. Macinko J, Guanais F, Souza M. Evaluation of the impact of Family Health Program on infant mortality in Brazil, 1990-2002. J Epidemiol Community Health 2006; 60:13-9.

7. Aquino R, Oliveira N, Barreto M. Impact of the family health program on infant mortality in Brazilian municipalities. Am J Public Health 2008; 99:87-93.

8. Bezerra Filho JG, Kerr LRFS, Miná DL, Barreto ML. Distribuição espacial da taxa de mortalidade infantil e principais determinantes no Ceará/Brasil, no período 2000-2002. Cad Saúde Pública 2007; 23:1173-85. 
9. Facchini L, Piccini R, Tomasi E, Silveira E, Siqueira F, Rodrigues M. Desempenho do PSF no Sul e no Nordeste do Brasil: avaliação institucional e epidemiológica da Atenção Básica à Saúde. Ciênc Saúde Coletiva 2006; 11:669-81.

10. Piccini R, Facchini L, Tomasi E, Thumé E, Silveira D, Siqueira F, et al. Efetividade da atenção pré-natal e de puericultura em unidades básicas de saúde do Sul e do Nordeste do Brasil. Rev Bras Saúde Matern Infant 2007; 7:75-82.

11. Roncalli A, Lima K. Impacto do PSF sobre indicadores de saúde da criança em municípios de grande porte do Nordeste do Brasil. Ciênc Saúde Coletiva 2006; 11:713-24.

12. Rocha PM, Uchoa AC, Rocha NSPD, Souza ECF, Rocha ML, Pinheiro TXA. Avaliação do Programa Saúde da Família em municípios do Nordeste brasileiro: velhos e novos desafios. Cad Saúde Pública 2008; 24 Suppl 1:S69-78.

13. Starfield B. Atenção primária: equilíbrio entre necessidades de saúde, serviços e tecnologia. Brasília: Organização das Nações Unidas para a Educação, a Ciência e a Cultura/Ministério da Saúde; 2002.

14. Chomatas E. Avaliação da presença e extensão dos atributos da atenção primária na rede básica de saúde no município de Curitiba [Dissertação de Mestrado]. Porto Alegre: Universidade Federal do Rio Grande do Sul; 2008.

15. Cunha C. Percepção da qualidade da atenção à saúde infantil pelos médicos e enfermeiros: comparação entre o programa saúde da família e o modelo tradicional [Dissertação de Mestrado]. Porto Alegre: Universidade Federal do Rio Grande do Sul; 2007.

16. Ibañez N, Rocha J, Castro P, Ribeiro M, Forster A, Novaes M, et al. Avaliação do desempenho da atenção básica no estado de São Paulo. Ciênc Saúde Coletiva 2006; 11:683-703.

17. Macinko J, Almeida C, Oliveira E, Sá P. Organization and delivery of primary health care services in Petrópolis, Brazil. Int J Health Plann Manage 2004; 19:303-17.

18. van Stralen CJ, Belisário SA, van Stralen TBS, Lima AMD, Massote AW, Oliveira CL. Percepção dos usuários e profissionais de saúde sobre atenção básica: comparação entre unidades com e sem saúde da família na Região Centro-Oeste do Brasil. Cad Saúde Pública 2008; 24 Suppl 1:S148-58.

19. Farias E, Stein A, Hoffmann J. Os egressos da residência de medicina de família e comunidade do Centro de Saúde-Escola Murialdo, 1976-2000. Bol Saúde 2004; 18:99-111.
20. Gerência de Saúde Comunitária, Grupo Hospitalar Conceição. Programa de residência médica em medicina de família e comunidade. Porto Alegre: Grupo Hospitalar Conceição; 2010.

21. Harzheim E, Duncan B, Stein A, Cunha C, Goncalves M, Trindade T, et al. Quality and effectiveness of different approaches to primary care delivery in Brazil. BMC Health Serv Res 2006; 5:156.

22. Departamento de Atenção Básica, Secretaria de Atenção em Saúde, Ministério da Saúde. Manual do instrumento de avaliação da atenção primária à saúde: Primary Care Assessment Tool. Brasília: Ministério da Saúde; 2010.

23. Brown H, Prescott R. Applied mixed models in medicine. 2nd Ed. Chichester: John Wiley \& Sons; 2006.

24. Machado M. Perfil dos médicos e enfermeiros de saúde da família no Brasil. http://www.ensp.fio cruz.br/psf_perfil/index.html (acessado em 12/ Ago/2009).

25. Tomasi E, Facchini LA, Piccini RX, Thumé E, Silveira DS, Siqueira FV, et al. Perfil sócio-demográfico e epidemiológico dos trabalhadores da atenção básica à saúde nas regiões Sul e Nordeste do Brasil. Cad Saúde Pública 2008; 24 Suppl 1:S193-201.

26. Kolling J. Orientação à atenção primária à saúde das equipes de saúde da família nos municípios do projeto telessaúde RS: estudo de linha de base [Dissertação de Mestrado]. Porto Alegre: Universidade Federal do Rio Grande do Sul; 2008.

27. Pimenta A, Coimbra A, Livorato F, Barros J, Rezende T. Pesquisa sobre organização e desempenho das Unidades de Saúde da Família de Amparo (SP): utilização de metodologia de avaliação rápida. Divulg Saúde Debate 2008; 42:102-17.

28. Elias PE, Ferreira CW, Alves MCG, Cohn A, Kishima V, Escrivão Junior A, et al. Atenção básica em saúde: comparação entre PSF e UBS por estrato de exclusão social no município de São Paulo. Ciênc Saúde Coletiva 2006; 11:633-41.

29. Serapioni M. Avaliação da qualidade em saúde: delineamentos para um modelo multidimensional e correlacional. In: Bosi ML, Mercado F, editores. Avaliação qualitativa de programas de saúde: enfoques emergentes. Petrópolis: Editora Vozes; 2006. p. 207-27.

30. Atkinson SJ. Anthropology in research on the quality of health services. Cad Saúde Pública 1993; 9:283-99.

Recebido em 16/Jul/2011

Versão final reapresentada em 11/Jun/2012

Aprovado em 18/Jun/2012 